\title{
VRestaurant: Challenges and opportunities in developing immersive projects in times of social distance
}

\author{
Keywords \\ Design, Electronic Art, Installation, Teaching, Virtual Reality.
}

VRestaurant is an installation of electronic art, design, gastronomy and multi-sensoriality. Its objective is to explore the potential of virtual reality (VR) to discuss issues related to healthy eating and sustainability. In it, we articulate academic research, linked to the Electronic Art Laboratory at PUC-Rio, with design project development practices, to achieve, through experimentation, an innovative result that contributes to changing people's behavior towards the future of food on the planet. For this, we searched for conceptions of experiences in VR, as well as "teaching-learning" methodologies (ALVES, 2008) in Design. In the methodology we use theoretical, field research, brainstorming and development. We work in a cyclical format, where each step is evaluated and validated. In the conceptual field, we ask: In 2050 will we be able to feed 10 billion people in a healthy and sustainable way to the planet? Humans produce food on a scale that has affected the climate and diverse ecosystems. The conversion of forests to pastures harms the migration of birds and alters the rain and planting periods. The foods produced are often of low nutritional value, which leads to unhealthy diets, causing malnutrition and obesity. Therefore, through awareness, engagement and emotion, we provide participants with a reflection that will stimulate changes in individual behavior, which can generate broader social changes. In the field of Design, we chose VR and its immersiveness, interactivity, multi-sensoriality and sense of presence, to provoke in the user a concrete experience. These areas are capable of producing emotional reactions in human beings. The feeling of experiencing an unfamiliar environment can be a pleasant experience for some and a difficult one for others. However, these sensations added to VR are invariably strong and impactful (SPITZ, 2021). With the restrictions imposed by SarsCov-2, the activities were directed to our homes, bringing a reality mediated by screens, which can be exhausting. Immersive technologies make these activities and contact with other people more enjoyable. Interactive possibilities also provide greater collaboration in task development. These potential allies to remote communication were the guiding points of VRestaurant. The development team is multidisciplinary, made up of professors, graduate students, undergraduates, professionals in design, gastronomy, arts, law, architecture, software engineering and cinema. By ensuring that each area is dealt with by those who have expertise, or who show interest in going deeper, even without experience. In our processes, we adapt the universe of VR headsets, through schematic representations, storyboards, videos, animations and text documents, always shared for everyone's analysis and consideration. The result of the process observed in the team is partial, but we conclude that in this multiplicity of profiles, origins and ages, there is a rich exchange of knowledge where subject " $A$ " learns and teaches simultaneously with subject " $B$ ". This approach, which prioritizes horizontal relationships (FREIRE, 1987), generated a vast theoretical, experimental and practical repository, in addition to an environment for creative development. Our academic and technical contribution demonstrates that working on the creation of VRestaurant also represented emotional support for the team in times of pandemic. 